\title{
Direct numerical simulation of solidifying liquid turbulence using the phase-field model
}

\author{
Takashi OHTA*, Takafumi ICHIYANAGI** and Taisei TANAKA** \\ * Department of Mechanical Engineering, University of Fukui \\ 3-9-1 Bunkyo, Fukui 910-8507, Japan \\ E-mail: t-ohta@u-fukui.ac.jp \\ ** Department of Mechanical Engineering, University of Fukui \\ 3-9-1 Bunkyo, Fukui 910-8507, Japan
}

Received: 30 June 2020; Revised: 31 August 2020; Accepted: 4 November 2020

\begin{abstract}
We realized a direct numerical simulation (DNS) of the turbulent flow of liquid along a solid wall with solidification by incorporating the phase-field model. The combination of DNS and phase-field model can clarify the mechanism of modulation of a turbulent boundary layer of liquid solidifying upon a solid wall and assist in constructing a prediction method in the future. The simulations allow observation of turbulent flow along a solid wall surface that grows with the solidification of a flowing liquid under an undercooling condition. In the flow field, turbulence structures such as velocity streaks and quasi-streamwise vortices were noted to diminish, and the turbulent flow tended to be laminar. In contrast, there were no changes in the turbulence statistics in the region above the growing solid-liquid interface. The solidification structure had a bent shape, which was caused by the effects of advection downstream and growing in the upstream direction owing to the undercooled fluid flowing from upstream. The wall surface grew non-uniformly depending on the local flow patterns and temperature distribution caused by turbulence structures close to the wall surface. The complex shape of the wall surface, which was observed during simulation, was originally triggered by the initial distribution of the turbulence structures. Sweep events in the highspeed streaks relatively expedited the growth of the solidification structures, which then modified the turbulence structures. The interaction between the turbulence structure and solidification structure promotes laminarization of the fluid flow.
\end{abstract}

Keywords : Turbulence structure, Solidification structure, Phase change, Turbulent flow, Direct numerical simulation, Phase-field model

\section{Introduction}

The phenomenon of undercooling, in which the phase changes from liquid to solid, and dendrite-like solidification structures grow, is of interest from the viewpoints of fluid dynamics, thermodynamic efficiency, and material mechanical strength. For example, this phenomenon can be observed at the interface between ice and water flowing on the ice surface, or inside the molten pool during welding. Moreover, when a liquid is flowing, a boundary layer is formed near the solid wall, and like many engineering-related flows, the flow field is likely to be a turbulent boundary layer. To predict such a flow field, it is necessary to consider the interaction between the solidification structure that solidifies and the flowing fluid; each of these is simultaneously affected by the other. To investigate the residual stresses that can exist in the solidified phase after welding, it is necessary to observe the relationship between the flow characteristics and the shape of the solidification structure in detail. Although it is difficult to directly observe the unsteady flow with solid-liquid phase change, it is desirable to reproduce the phenomenon by a numerical simulation and observe the results in detail.

For example, the volume of fluid (VOF) method (Hirt and Nichols, 1981) has been used to calculate melting and solidification in a molten pool of arc welding (Wang and Tsai, 2001). Assuming that such a molten pool actually exhibits turbulent flow, a simulation of a three-dimensional turbulent molten pool for gas metal arc welding was performed using 
the $k$ - $\varepsilon$ model (Park et al., 2019). However, the $k-\varepsilon$ model is not suitable for detailed observations of the turbulence and solidification structures because the complexity of the interface geometry renders the interfacial advection and construction difficult. In addition, when using interface tracking methods, such as the VOF method and immersed boundary method (Peskin, 2002; Mittal and Iaccarino, 2005), to represent the motion of the interface, we need to introduce a physical model to calculate the phase change and interfacial tension (Karagadde et al., 2012), which makes the calculations more complicated.

The phase-field model (Kobayashi, 1993; Kobayashi, 1994; Boettinger et al., 2002; Takaki, 2014) can be used to simultaneously predict the interfacial movement and phase change. In the phase-field model, a time-evolution equation for a phase-field variable is solved, which allows us to track the interface with a single threshold. The construction of the interface and the interfacial tension can then be introduced into the time-evolution equation by means of a function consisting of the double-well potential and the gradient square term. This method can reproduce the growth of solidification structures such as dendrites. Therefore, it is considered useful to combine the model with a direct numerical simulation (DNS), which allows detailed observation of turbulence phenomena. Thus far, as an example of combining this method with a numerical simulation of fluid flow, a simulation considering the interaction between fluid and precipitation has been performed (Hawkins et al., 2013; Hawkins et al., 2014).

In this study, we demonstrate the feasibility of reproducing a turbulent boundary layer with solid-liquid phase change under an undercooling condition using DNS to aid the phase-field model for observing the characteristics of the turbulent boundary layer of the solidifying liquid. Specifically, we assumed that water was flowing along an ice wall and observed the relationship between the turbulence characteristics and progress of solidification to clarify the qualitative mechanism of interaction between turbulence modulation and solidification formation.

\section{Simulation method}

\subsection{Fundamental equations for fluid flow}

In this study, DNS was performed using the fundamental equations for three-dimensional incompressible flows.

Continuity equation:

$$
\frac{\partial u_{j}}{\partial x_{j}}=0
$$

Navier-Stokes equation:

$$
\frac{\partial u_{i}}{\partial t}+u_{j} \frac{\partial u_{i}}{\partial x_{j}}=-\frac{1}{\rho} \frac{\partial p}{\partial x_{i}}+\frac{1}{\rho} \frac{\partial}{\partial x_{j}} \mu\left(\frac{\partial u_{i}}{\partial x_{j}}+\frac{\partial u_{j}}{\partial x_{i}}\right)+X_{i},
$$

where $x_{i}(i=1,2,3)$ are the spatial coordinates, $u_{i}$ are the velocity components, $t$ is the elapsed time, $p$ is the pressure, $\rho$ is the fluid density, $\mu$ is the viscosity, and $X_{i}$ is the force acting on the fluid and affecting it. These equations are expressed according to the Einstein summation convention with respect to the index $j$.

\subsection{Phase-field model}

A phase-field variable $\phi$ is defined for a simulation of a solid-liquid mixed-phase field with a phase change of $\phi=1$ in the solid phase and $\phi=-1$ in the liquid phase. The following equations are used as the fundamental equation of the phase-field model (Allen and Cahn, 1979; Boettinger et al., 2002; Takaki, 2014).

\section{Free energy functional:}

$$
F=\int_{V}\left(f_{\text {chem }}+f_{\text {doub }}+f_{\text {grad }}\right) \mathrm{d} V
$$

Allen-Cahn equation:

$$
\frac{D \phi}{D t}=-M_{\phi} \frac{\delta F}{\delta \phi}
$$

\section{Thermal conduction equation:}

$$
\frac{D T}{D t}=\frac{K}{C_{p}} \nabla^{2} T+\frac{1}{2} \frac{L}{C_{p}} \frac{\partial P(\phi)}{\partial t}
$$

Here, $M_{\phi}$ is the phase-field mobility, $T$ is the temperature, $K$ is the thermal conductivity, $L$ is the latent heat, $C_{p}$ is the specific heat, and $P(\phi)$ is the energy density function. The chemical free energy density $f_{\text {chem }}$, double-well potential energy $f_{\text {doub }}$, and gradient energy density $f_{\text {grad }}$ are expressed as follows:

$$
f_{\text {chem }}=\frac{1}{2}\left\{(1+P(\phi)) f_{\mathrm{S}}+(1-P(\phi)) f_{\mathrm{L}}\right\}
$$




$$
\begin{aligned}
& f_{\text {doub }}=W Q(\phi) \\
& f_{\text {grad }}=\frac{a^{2}}{2}|\nabla \phi|^{2}
\end{aligned}
$$

where $f_{\mathrm{S}}$ and $f_{\mathrm{L}}$ indicate the chemical free energy density of the solid and liquid phase, respectively, $W$ is the height of the energy barrier, and $a$ is the gradient coefficient. The energy density function $P(\phi)$ and double-well potential function $Q(\phi)$ are

$$
\begin{aligned}
& P(\phi)=\frac{15}{8}\left(\phi-\frac{2}{3} \phi^{3}+\frac{1}{5} \phi^{5}\right), \\
& Q(\phi)=\frac{1}{16}\left(1-\phi^{2}\right)^{2} .
\end{aligned}
$$

Using the melting temperature $T_{m}$, the energy density difference between the solid and liquid phases is expressed as

$$
f_{\mathrm{S}}-f_{\mathrm{L}}=\frac{L\left(T-T_{m}\right)}{T_{m}} .
$$

From the above equations, we obtain the time-evolution equation using the functional derivative in terms of the phase-field variable $\phi$ considering the anisotropy in Eq. (8) owing to the non-uniformity of the gradient coefficient $a$.

Time-evolution equation for the phase-field variable $\phi$ : (Takaki, 2009)

$$
\begin{gathered}
\frac{D \phi}{D t}=M_{\phi}\left[\nabla\left(a^{2} \nabla \phi\right)+\frac{\partial}{\partial x}\left(a \frac{\partial a}{\partial \phi_{, x}}|\nabla \phi|^{2}\right)+\frac{\partial}{\partial y}\left(a \frac{\partial a}{\partial \phi_{, y}}|\nabla \phi|^{2}\right)+\frac{\partial}{\partial z}\left(a \frac{\partial a}{\partial \phi_{, z}}|\nabla \phi|^{2}\right)\right. \\
\left.+\frac{1}{4} W\left(1-\phi^{2}\right)\left\{\phi-\frac{15}{4 W} \times\left(1-\phi^{2}\right) \frac{L\left(T-T_{m}\right)}{T_{m}}\right\}\right]
\end{gathered}
$$

where $\partial \phi_{, x}=\partial \phi / \partial x, \partial \phi_{, y}=\partial \phi / \partial y$, and $\partial \phi_{, z}=\partial \phi / \partial z$. The gradient coefficient with the anisotropy is

$$
a(\nabla \phi)=\bar{a}(1-3 \zeta)\left\{1+\frac{4 \zeta}{1-3 \zeta} \frac{\left(\phi_{, x}\right)^{4}+\left(\phi_{, y}\right)^{4}+\left(\phi_{, z}\right)^{4}}{|\nabla \phi|^{4}}\right\},
$$

where $\bar{a}$ and $\zeta$ indicate the average value of the gradient coefficient, and strength of the anisotropy, respectively.

The phase-field mobility $M_{\phi}$, standard value of the gradient coefficient $\bar{a}$, and height of the energy barrier $W$ are related to the physical property values as follows:

$$
M_{\phi}=\frac{4 b T_{m}}{3 \delta L} \eta, \quad \bar{a}=\frac{1}{2} \sqrt{\frac{3 \delta \gamma}{b}}, \quad W=\frac{6 \gamma b}{\delta}
$$

Here, $\delta, \eta$, and $\gamma$ denote the interface thickness, interfacial kinetic coefficient, and interfacial energy, respectively. The coefficient concerning the interface thickness, $b$, is defined as $b=\tanh ^{-1}\left\{2 \lambda /\left(1+\lambda^{2}\right)\right\}$, where $\lambda$ is a threshold that defines the interface width in the equilibrium profile of the phase-field variable. We set $\lambda=0.8$ based on the criterion employed in a past study (Takaki, 2009). The influence of the fluid flow on the phase-field variable is given by the convection velocity in the substantial derivative in Eq. (12) and the temperature distribution.

\subsection{Simulation conditions}

The first step in the present study is to produce a turbulent boundary layer of water along a wall, which is assumed to be ice. In the simulation, the axes in the streamwise, wall-normal, and spanwise directions are $x, y$, and $z$, and the velocity components are $u, v$, and $w$, respectively. We numerically solved the fundamental equations of the three-dimensional incompressible fluid flow (1)(2). For boundary conditions, we applied the non-slip condition to the wall surface and the free-slip condition to the outer boundary. The periodic boundary condition was used in the streamwise and spanwise directions to simulate a fluid flow virtually along an infinitely large wall. A constant volume force was continuously applied to maintain the flow. The lengths of the computational domain are $L_{x}=0.0103 \mathrm{~m}, L_{y}=0.00206 \mathrm{~m}$, and $L_{z}=0.00516 \mathrm{~m}$. We used a collocated grid system. The number of grid points is 64 in the streamwise and spanwise directions, and 128 in the wall-normal direction. The grid spacings, which were normalized with the friction velocity and kinematic viscosity at the wall surface, were $\Delta x^{+}=18, \Delta y^{+}=1.8$, and $\Delta z^{+}=9$. The resolution was determined mainly for the turbulent flow simulation to ensure the stability of simulation but not necessarily suited for the phase-field model. We validated the qualitative reliability of the simulation results pertaining to the phase-field model by comparisons 
Table 1 Parameters for water in the phase-field model

\begin{tabular}{l|l}
\hline Melting temperature $T_{m}$ & $273.15 \mathrm{~K}$ \\
Temperature of undercooling fluid $T_{0}$ & $272.15 \mathrm{~K}$ \\
Thermal conductivity $K$ & $0.561 \mathrm{~W} / \mathrm{m} \cdot \mathrm{K}$ \\
Specific heat $C_{p}$ & $4.22 \times 10^{6} \mathrm{~J} / \mathrm{K} \cdot \mathrm{m}^{3}$ \\
Latent heat $L$ & $8.00 \times 10^{6} \mathrm{~J} / \mathrm{m}^{3}$ \\
Strength of the anisotropy $\zeta$ & 0.03 \\
Interface thickness $\delta$ & $3 \Delta y$ \\
Interface kinetic coefficient $\eta$ & $2.0 \mathrm{~m} / \mathrm{K} \cdot \mathrm{s}$ \\
Interface energy $\gamma$ & $0.37 \mathrm{~J} / \mathrm{m}^{2}$ \\
\hline
\end{tabular}

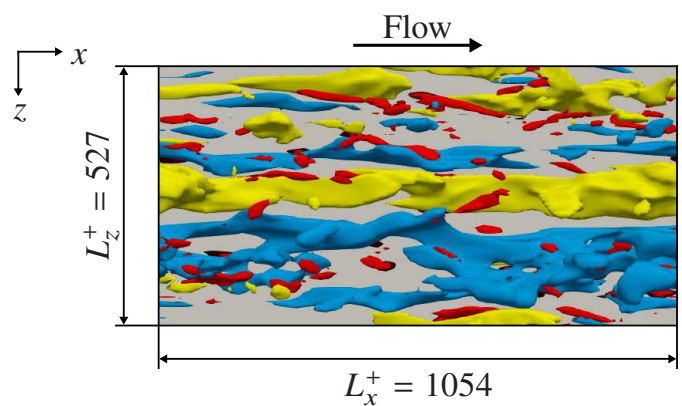

Fig. 1 Instantaneous distributions of high- and low-speed regions and quasi-streamwise vortices (yellow: $u^{++}=3$, blue: $u^{\prime+}=-3$, red: $\left.\omega_{x}^{+}= \pm 0.3\right)$

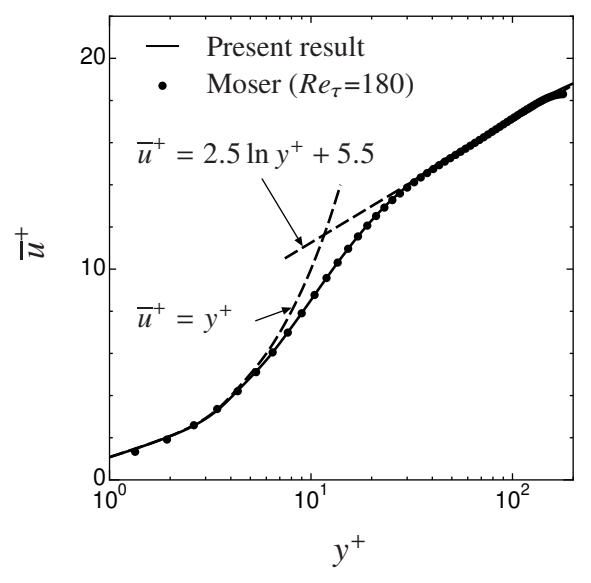

Fig. 2 Profile of the temporally and spatially averaged streamwise velocity

with those obtained using isotropic resolution. The numerical methods used herein are the same as those mentioned below. At this time, we could simulate a flow with Reynolds number $R e=\rho U L_{y} / \mu_{\mathrm{L}}=3966$ and Prandtl number $\operatorname{Pr}=\mu_{\mathrm{L}} C_{p} / \rho K=13.47$, where $U$ was the mean velocity at $y=L_{y}$.

After the turbulence was fully developed and the flow field reached a quasi-steady state, the phase-field model was activated under an undercooling condition to simulate the turbulent boundary layer of solidifying water. To accomplish this, we numerically solved the fundamental equations for the three-dimensional incompressible fluid flow (1)(2), the heat conduction equation (5), and the time-evolution equation for the phase-field variable (12). The time marching was performed using the fractional step method, in which the 2nd-order Adams-Bashforth method was applied to the convection, viscous, and external force terms in the Navier-Stokes equation (2), and the backward Euler method was applied to the pressure term and the continuity equation (1). In parallel, the thermal conduction equation and time-evolution equation of the phase-field variable are calculated using the forward Euler method. The spatial derivatives in the fundamental equations were approximated with the 4th-order central finite difference. For the convection terms, we used the difference method along with the conservation of energy (Kajishima et al., 1998) to realize DNS of turbulent flow without any numerical diffusion. The time increment was $\Delta t=10^{-8} \mathrm{~ms}$. At each time step, the pressure equation in the fractional step method was solved efficiently using the residual cutting method (Tamura et al., 1997).

Assuming the physical properties of water at temperature $T=0{ }^{\circ} \mathrm{C}$, the density is $\rho=999.84 \mathrm{~kg} / \mathrm{m}^{3}$. The parameters for the phase-field model are shown in Table 1. In this simulation, the latent heat $L$ is set to be smaller than the actual value, as proposed in a past study (Nakabeppu and Hijikata, 1998). As the effect of the phase-field model on the flow field, the viscosity was set as follows so that the viscosity $\mu$ changed depending on the phase-field variable $\phi$.

$$
\mu=\frac{1-P(\phi)}{2} \mu_{\mathrm{L}}+\frac{1+P(\phi)}{2} \mu_{\mathrm{S}}
$$

where $\mu_{\mathrm{L}}$ and $\mu_{\mathrm{S}}$ indicate the viscosities of the liquid phase $(\phi=-1)$ and solid phase $(\phi=1)$, respectively. This represents the viscosity of the multi-phase, resulting in the solid-liquid interface, as the weighted arithmetic mean of the viscosities of the liquid and solid phases, with the assumption that the viscous stress of the multi-phase is the sum of the viscous stresses of the liquid and solid phases for a common strain rate. The viscosity of water is $\mu_{\mathrm{L}}=0.0017906 \mathrm{~Pa} \cdot \mathrm{s}$ and, 


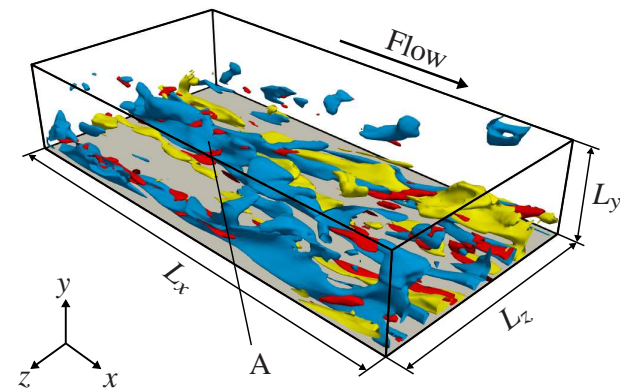

(a) $t=0$

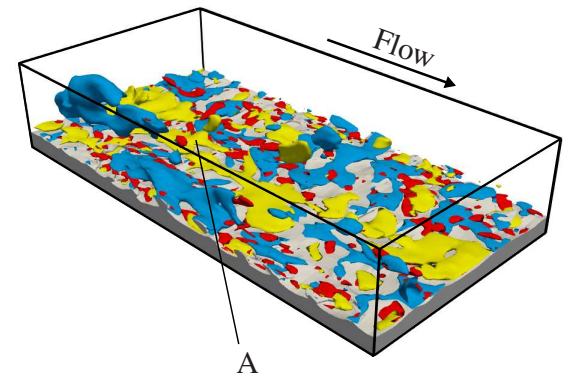

(b) $t=4.7 \mathrm{~ms}$

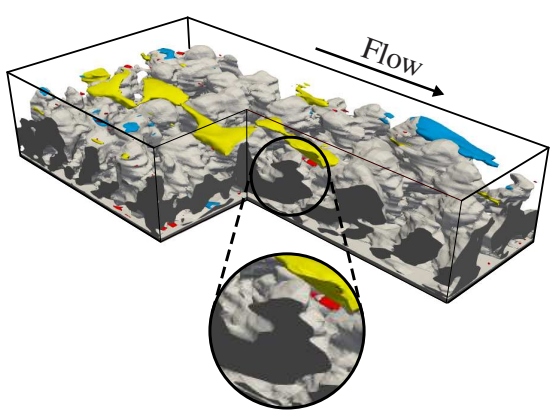

(c) $t=6.6 \mathrm{~ms}$

Fig. 3 Instantaneous distributions of high- and low-speed regions, quasi-streamwise vortices, and solid phase at each time (yellow: $u^{\prime+}=3$, blue: $u^{\prime+}=-3$, red: $\omega_{x}^{+}= \pm 0.3$, gray: $\phi \geq 0$ )

assuming that the viscosity of the solid phase is sufficiently large, the parameter $\mu_{\mathrm{S}}$ is set as $\mu_{\mathrm{S}}=100 \mu_{\mathrm{L}}$. Because we focus on the liquid flow and phase change, in the simulation, we assume that the density, thermal conductivity, and specific heat in the solid phase are constant and equal to those in the liquid phase.

As an initial condition, we set the liquid phase $(\phi=-1)$ in the entire flow field, and the solid phase $(\phi=1)$ on the wall surface. At one moment after turbulent flow was fully developed under the above conditions, we set the temperature distribution as

$$
T=T_{0}+\frac{\phi+1}{2}\left(T_{m}-T_{0}\right)
$$

and activated the phase-field model.

\subsection{Verification of fully developed turbulent boundary layer}

Figure 1 shows instantaneous turbulence structures as a result of simulating a turbulent boundary layer using only the fundamental equations for three-dimensional incompressible flow (1)(2) before starting a simulation with the phase-field model. The flow field is viewed from the top toward the wall, and the velocity streaks and quasi-streamwise vortices are visualized using isosurfaces of the streamwise velocity fluctuation $u^{\prime+}$ and streamwise vorticity $\omega_{x}^{+}$. The velocity streaks extending in the streamwise direction, which are a characteristic of a turbulent boundary layer, can be observed, as well as the quasi-streamwise vortices that exist between the high- and low-speed streaks. Due to the size constraint of the computational domain and the periodic boundary condition, the streaks are connected to themselves in the streamwise direction. Nevertheless, the streamwise mean velocity profile shown in Fig. 2 is consistent with the wall law $\bar{u}^{+}=y^{+}$and logarithmic law $\bar{u}^{+}=2.5 \ln y^{+}+5.5$, which are features of a developed turbulent boundary layer. Furthermore, the profile is also consistent with a previous DNS result (Moser et al., 1999). These proved that a fully developed turbulent boundary layer was produced in the simulation.

\section{Relationship between turbulence modulation and solidification structure growth}

\subsection{Features of turbulent flow of solidifying liquid}

We set the time to $t=0$ at the moment that the phase-field model was activated in the simulation. Figure 3 shows the velocity streaks and quasi-streamwise vortices in the flow field at each time while the undercooled fluid is solidifying. A flow field in which the solid-liquid interface grows from the relatively hot wall toward the undercooled fluid over time was simulated. 


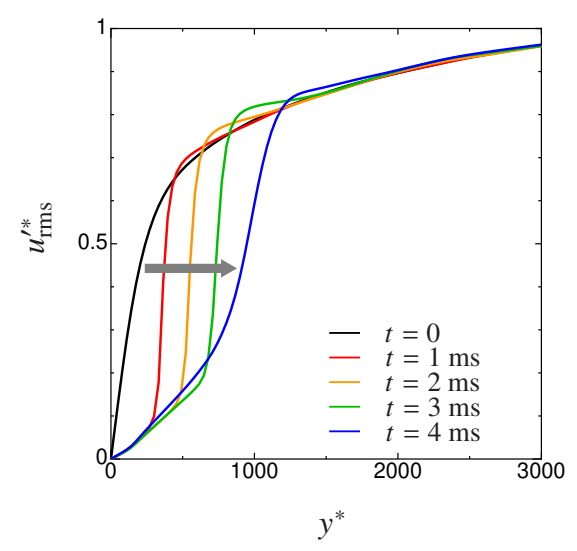

Fig. 4 Profiles of the streamwise mean velocity at each time

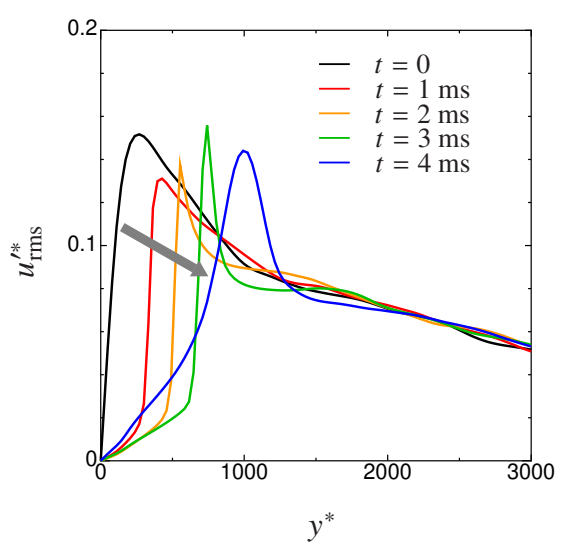

Fig. 5 Profiles of the streamwise velocity fluctuation intensity at each time

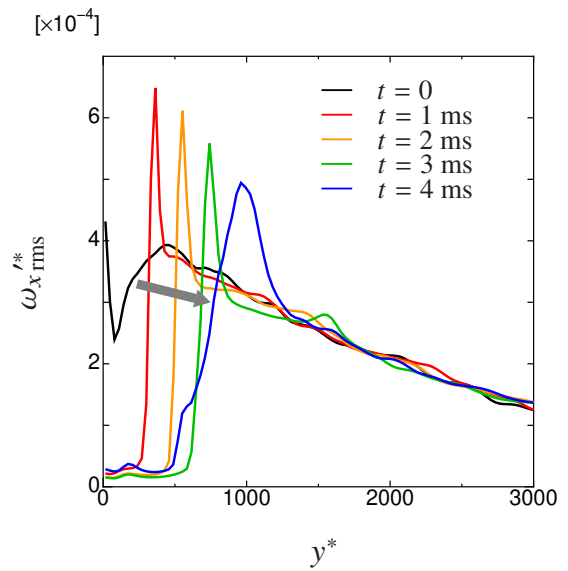

Fig. 6 Profiles of the streamwise vorticity fluctuation intensity at each time

By focusing on the growth of the solid phase, we found that at $t=0$, the solid phase had a smooth surface shape, but at $t=4.7 \mathrm{~ms}$, it grew to undulate in the streamwise direction, and at $t=6.6 \mathrm{~ms}$, it had grown into a bent shape. When the shape of the solidification structure is somewhat small, it is advected downstream following the flow. After a certain amount of growth, only the tip of the structure grows in the upstream direction in contact with the undercooled fluid flowing from upstream. The feature that the solidification structure grows upstream is consistent with the result of a precipitation simulation (Hawkins et al., 2013; Hawkins et al., 2014), which has been pointed out to be mathematically similar to undercooled solidification.

The velocity streaks and quasi-streamwise vortices disappear as the solid surface grows with time, suggesting that the flow field is transitioning to a laminar flow state. Meanwhile, at $t=0$, the low-speed region shown in blue is located at A, whereas, at $t=4.7 \mathrm{~ms}$, the high-speed region shown in yellow exists at the same location. This means that the mechanism where the turbulence structure is changed as solidification progresses may be at work.

\subsection{Modulation of turbulence statistics}

From the viewpoint of turbulence statistics, we investigate the relationship between the turbulence and solidification structure. In this and the following sections, the results of the turbulence statistics are shown spatially averaged in the $x-z$ directions in the instantaneous flow field at each time. Because the physical properties on the wall change drastically as the solidification structure grows, instead of the wall friction velocity $u_{\tau}$ and kinematic viscosity $v_{\mathrm{w}}$ of the fluid flowing on the wall, the statistics are normalized using the main flow velocity $u_{\infty}$ and mainstream kinematic viscosity $v_{\infty}$, and are indicated by the superscript $*$. Figure 4 shows the profiles of the streamwise mean velocity. As time is elapsing and the solid-phase surface is growing, the viscosity increases owing to solidification and the velocity decreases in the solid 


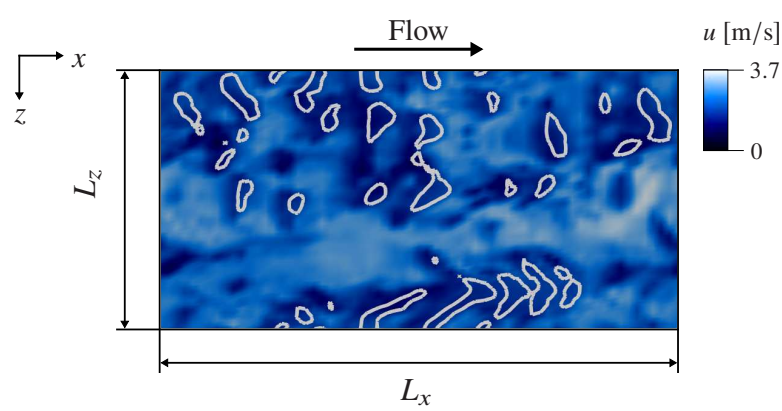

Fig. 7 Instantaneous distributions of streamwise velocity and solid-liquid interface on an $x-z$ plane of $y^{*}=800$ at $t=3 \mathrm{~ms}$

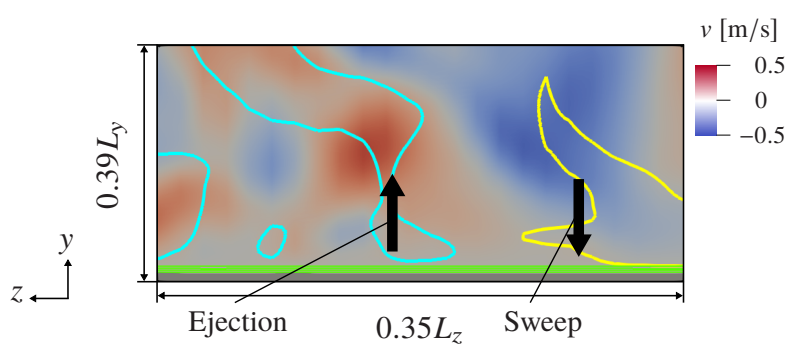

(a) $t=0.5 \mathrm{~ms}$

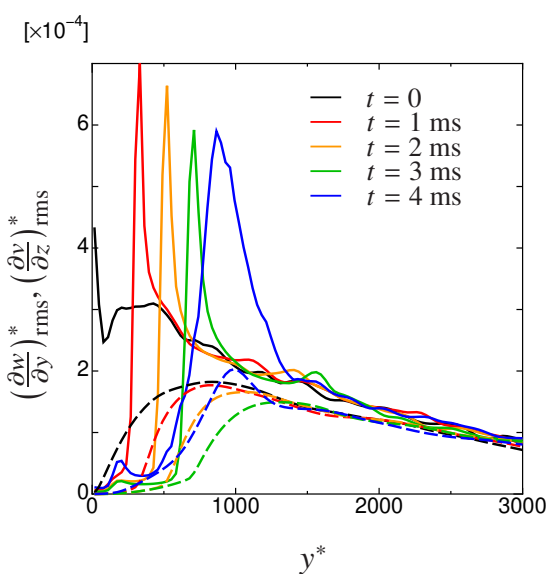

Fig. 8 Profiles of the root mean square of each component of streamwise vorticity at each time

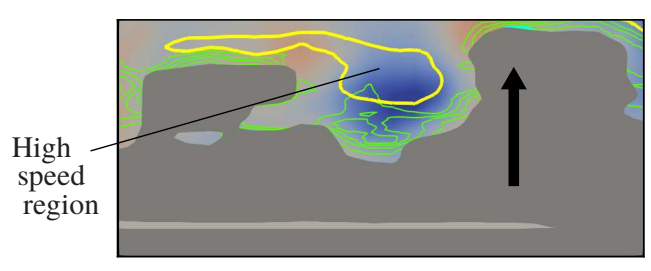

(b) $t=2.3 \mathrm{~ms}$

Fig. 9 Instantaneous distributions of high- and low-speed regions (yellow: $u^{\prime+}=2$, blue: $u^{\prime+}=-2$ ), solid phase (gray: $\phi \geq 0$ ), wall-normal velocity, and isolines of temperature on a $y-z$ plane of $x / L_{x}=0.44$ at each time

phase. Because there is no change observed in the region above the solid-liquid interface, the solidification velocity is higher than the viscous diffusion velocity in the present simulation of solidification under the undercooling condition.

In order to statistically investigate the disappearance of the turbulence structures shown in Fig. 3, the profiles of the streamwise velocity fluctuation intensity and streamwise vorticity fluctuation intensity are shown in Figs. 5 and 6 , respectively. As the solid phase grows, the velocity and vorticity fluctuation intensities become smaller in the solid phase, whereas they do not change in the region above the solid-liquid interface. At $t=1 \mathrm{~ms}$, owing to the increase in viscosity caused by the growth of the solid phase, the velocity fluctuation intensity is reduced. After $t=2 \mathrm{~ms}$, there is a portion where the velocity fluctuation intensity is greater than that before the solidification started. In addition, the vorticity fluctuation intensity becomes larger after $t=1 \mathrm{~ms}$ than that before the solidification started. Because the timings at which the tendencies of the velocity and vorticity fluctuation intensities are different, the mechanisms of the changes are considered to be different.

After $t=2 \mathrm{~ms}$, the dendritic solid phase grew, and both the solid and liquid phases existed in a common $x-z$ plane. This caused a large difference in the flow velocity in the plane, and apparently increased the velocity fluctuation intensity. As an example, Fig. 7 shows the distribution of the streamwise velocity and the solid-liquid interface in the $x-z$ plane of $y^{*}=800$ at $t=3 \mathrm{~ms}$. The flow field is viewed from the top toward the wall. The blue distribution indicates the streamwise velocity $u$ and the gray lines indicate the solid-liquid interface $(\phi=0)$ in the plane. The velocity is small in the region surrounded by the gray lines, that is, in the solid phase, and the fluid is flowing in the liquid phase region. This means that the increase in the velocity fluctuation intensity did not originate from the turbulence phenomenon, but from the solid-liquid multi-phase.

In order to investigate the reason why the vorticity fluctuation intensity becomes partially large in some cases, Fig. 8 shows the profiles of each term of the vorticity $\omega_{x}=\partial w / \partial y-\partial v / \partial z$. The solid and dashed lines indicate the intensities of the terms $\partial w / \partial y$ and $\partial v / \partial z$, respectively. Strong shear $\partial w / \partial y$ is generated at the interface while the solid-liquid interface is growing. Because the shear at the interface has the same tendency as the shear of the wall at $t=0$, it is considered that 


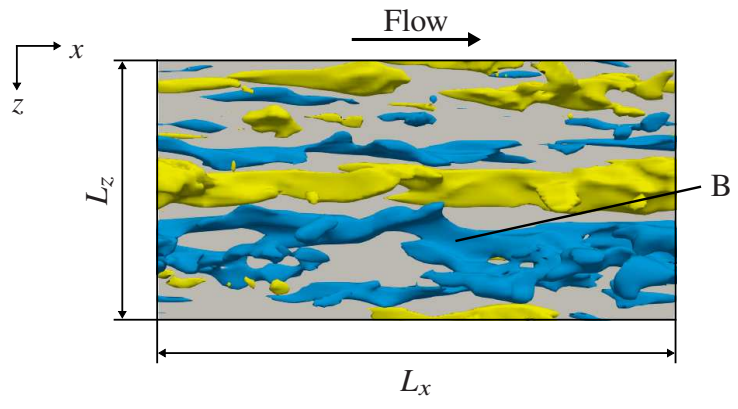

(a) High- and low-speed regions $(t=0)$

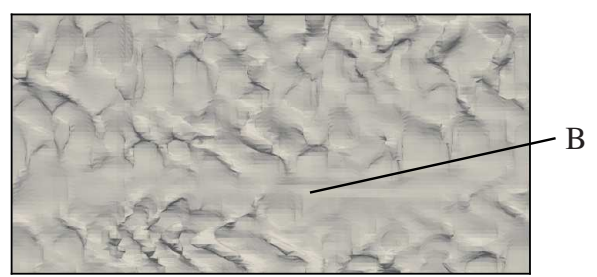

(b) Solid surface $(t=2.5 \mathrm{~ms})$

Fig. 10 Instantaneous distributions of high- and low-speed regions and solid phase at each time (yellow: $u^{\prime+}=3$, blue: $u^{++}=-3$, gray: $\phi \geq 0$ )

the growing interface plays the same role as that of the wall surface. From the observation of the instantaneous turbulence structure and the turbulence statistics obtained from the DNS, it was found that although there are some changes specific to the phase change at the growing interface, the turbulence was suppressed near the solid-liquid interface as solidification progressed, and no effect was seen in the region above the interface.

\subsection{Influence of turbulence structure on solidification}

To investigate the formation mechanism of the solidified wall shape and the reason for the exchange between the low- and high-speed regions before and after solidification, we observed the turbulence structure near the wall. Figure 9 shows the feature in a $y$ - $z$ plane at each time. The flow field is viewed in the streamwise direction, and the fluid flows from the back to the front. The yellow and blue lines indicate $u^{\prime+}=2$ and $u^{\prime+}=-2$, respectively, the gray region indicates the solid phase $\phi \geq 0$, the red and blue distribution represents the wall-normal component of the velocity, $v$, and the green lines indicate the isolines of the temperature. At $t=0.5 \mathrm{~ms}$, the surface of the solid phase is parallel to the initial wall surface, and the temperature contours are also parallel. After that, however, the temperature gradient is relatively large just below the high-speed streak shown in yellow, due to the effect of sweep, which also causes the high-speed region to fall. In contrast, the temperature gradient is relatively small just below the low-speed streak, due to the effect of ejection, which also causes the low-speed region to rise. As shown in Eq. (11), the difference in energy density between the solid and liquid phases, which is the chemical driving force for solidification of pure substance, is proportional to $T-T_{m}$. Therefore, the growth rate is higher just below the high-speed streaks and lower below the low-speed streaks. Then, at $t=2.3 \mathrm{~ms}$, valleys are formed in the part where the low-speed streaks existed, and the fluid flows more easily than in the surroundings.

Figure 10 shows the distribution of the velocity streaks and the surface of solid phase in the flow field, which is viewed from the top. For example, at point B where there was a low-speed streak at $t=0$, a valley of the solidification structure was generated at $t=2.5 \mathrm{~ms}$, and the fluid flowed more easily, resulting in a high-speed region. As a result, it was found that the solidification structure was generated in accordance with the distribution of the instantaneous turbulence structure, and the turbulence structure changed according to the distribution of the solidification structure. This interaction selectively suppresses the high-speed flow and promotes laminarization of the flow field.

\section{Conclusions}

In this study, it was shown that a turbulent boundary layer with solid-liquid phase change can be simulated by combining DNS of the turbulent flow and the phase-field model. Furthermore, by observing the results, we found the following.

- The turbulence statistics showed that the turbulence became weaker as the solid-liquid interface grew toward the flow field. The solid-liquid interface played the same role as that of the wall surface.

- From the observation of the instantaneous structure in the flow field, the disturbance of the turbulent flow generated the initial solidification structure. Next, regarding shape of the solidification structure, the wall shape grew toward the upstream while advancing to the downstream by the main flow.

- A distribution of the solidification structure was generated that corresponded to the instantaneous turbulence structure near the wall. The turbulence structure then changed, owing to the generated solidification structure, which promoted 
laminarization near the wall surface.

Although the results from the phase-field model, unlike DNS of flow, may depend on the grid resolution and interface width, the above conclusions are qualitatively invariant for the different conditions. From the results of this simulation, we can expect to develop a turbulent flow model that can predict this turbulent flow modulation, and predict the residual stress that exists after solidification.

\section{References}

Allen, S. M. and Cahn, J. W., A microscopic theory for antiphase boundary motion and its application to antiphase domain coarsening, Acta Metallurgica, Vol.27, No.6 (1979), pp.1085-1095.

Boettinger, W. J., Warren, J. A., Beckermann, C. and Karma, A., Phase-field simulation of solidification, Annual Review of Materials Research, Vol.32, No.1 (2002), pp.163-194.

Hawkins, C., Angheluta, L., Hammer, Ø. and Jamtveit, B., Precipitation dendrites in channel flow, EPL (Europhysics Letters), Vol.102, No.5 (2013), pp.54001.

Hawkins, C., Angheluta, L. and Jamtveit, B., Hydrodynamic shadowing effect during precipitation of dendrites in channel flow, Physical Review E, Vol.89, No.2 (2014), pp.022402.

Hirt, C. W. and Nichols, B. D., Volume of fluid (VOF) method for the dynamics of free boundaries, Journal of Computational Physics, Vol.39, No.1 (1981), pp.201-225.

Kajishima, T., Ohta, T., Okazaki, K. and Miyake, Y., High-order finite-difference method for incompressible flows using collocated grid system, JSME International Journal Series B Fluids and Thermal Engineering, Vol.41 (1998), pp.830839.

Karagadde, S., Bhattacharya, A., Tomar, G. and Dutta, P., A coupled VOF-IBM-enthalpy approach for modeling motion and growth of equiaxed dendrites in a solidifying melt, Journal of Computational Physics, Vol.231, No.10 (2012), pp.3987-4000.

Kobayashi, R., Modeling and numerical simulations of dendritic crystal growth, Physica D: Nonlinear Phenomena, Vol.63, No.3-4 (1993), pp.410-423.

Kobayashi, R., A numerical approach to three-dimensional dendritic solidification, Experimental Mathematics, Vol.3, No.1 (1994), pp.59-81.

Mittal, R. and Iaccarino, G., Immersed boundary methods, Annual Review of Fluid Mechanics, Vol.37 (2005), pp.239261.

Moser, R. D., Kim, J. and Mansour, N. N., Direct numerical simulation of turbulent channel flow up to $R e_{\tau}=590$, Physics of Fluids, Vol.11, No.4 (1999), pp.943-945.

Nakabeppu, O. and Hijikata, K., Simulation of supercooling solidification by phase field model, Transactions of the Japan Society of Mechanical Engineers, Series B, Vol.64, No.618 (1998), pp.463-470. (in Japanese)

Park, K., Jeong, H., Baek, S., Kim, D.-Y. Kang, M.-J. and Cho, J, Turbulent molten pool analysis of tandem GMA automotive steel sheet welding, International Journal of Heat and Mass Transfer, Vol.129 (2019), pp.1-6.

Peskin, C. S., The immersed boundary method, Acta Numerica, Vol.11 (2002), pp.479-517.

Takaki, T., Science of machine (Kikai no Kenkyu), Yokendo, Vol.61, No.9 (2009), pp.921-926. (in Japanese)

Takaki, T., Phase-field modeling and simulations of dendrite growth, ISIJ International, Vol.54, No.2 (2014), pp.437-444.

Tamura, A., Kikuchi, K. and Takahashi, T., Residual cutting method for elliptic boundary value problems: Application to Poisson's equation, Journal of Computational Physics, Vol.137, No.2 (1997), pp.247-264.

Wang, Y. and Tsai, H. L., Impingement of filler droplets and weld pool dynamics during gas metal arc welding process, International Journal of Heat and Mass Transfer, Vol.44, No.11 (2001), pp.2067-2080. 